\section{PSICOLOGIA IBEROAMERICANA}

\section{Psicología lberoamericana}

ISSN: 1405-0943

psicología.iberoamericana@uia.mx

Universidad Iberoamericana, Ciudad de

México

México

Tovar-Hernández, Deysy Margarita; Rocha Sánchez, Tania Esmeralda Masculinidades: espacios-momentos críticos en las formas de ser hombre en la Ciudad de México

Psicología Iberoamericana, vol. 20, núm. 2, julio-diciembre, 2012, pp. 7-15

Universidad Iberoamericana, Ciudad de México

Distrito Federal, México

Disponible en: http://www.redalyc.org/articulo.oa?id=133928816002

Cómo citar el artículo

Número completo

- Más información del artículo

- Página de la revista en redalyc.org



Sistema de Información Científica

Red de Revistas Científicas de América Latina, el Caribe, España y Portugal

Proyecto académico sin fines de lucro, desarrollado bajo la iniciativa de acceso abierto 


\title{
Masculinidades: espacios-momentos críticos en las formas de ser hombre en la Ciudad de México
}

\author{
Masculinities: Critical Periods in the Way that Mexican Adults \\ Define what it Means to be a Man
}

\author{
Deysy Margarita Tovar-Hernández* \\ Tania Esmeralda Rocha Sánchez \\ FACULTAD de PSICOLOGía \\ Universidad Nacional Autónoma de México
}

\section{RESUMEN}

El propósito de este artículo es analizar las formas en que los hombres están construyendo sus masculinidades alrededor del "modelo hegemónico", en el contexto actual de cambios socioculturales y transiciones en el rol de género. Por lo anterior, se realizó un estudio exploratorio y cualitativo; se aplicaron 50 cuestionarios abiertos a hombres de la Ciudad de México (edad promedio = 27.8), y con la información obtenida se llevó a cabo un análisis de contenido para delimitar el tipo de masculinidades reconocidas por los varones y sus características. Posteriormente se hizo un análisis de tales hallazgos para identificar el momento de transición y posicionamiento de estas masculinidades en una suerte de continuo que va desde lo tradicional hasta lo contracultural. En los discursos de los participantes se reconocen masculinidades emergentes que apuestan al ideal de "equidad de género" y la autonomía individual, al mismo tiempo que prevalecen formas tradicionales que se conectan con diferentes prácticas no equitativas. Estos patrones tienen un impacto importante en el contexto de las relaciones interpersonales.

Palabras clave: masculinidades, hombres, transformaciones sociales, transición del rol de género.

\section{ABSTRACT}

The main aim of this article is to understand how Mexican men are living their masculinities in the context of different gender role transitions and sociocultural changes. To explore this issue, our analytical framework examines the different ways in which men are living or not their self construction around hegemonic masculinity, also we analyzed different positions across a theoretical continuum since traditional to alternative behaviors and traits. We work with 50 Mexican men $\left(_{X}=27.8\right.$ years old $)$ in an exploratory and qualitative study. The result shows a diversity of masculinity patterns, which are analyzed inside the theoretical view of gender role transformations; emerging new and equal masculinities in which men's practices closely match the ideals of gender equality and individual autonomy, as the same time that remains traditional masculinities that are connected to different forms of "gender unequal" practices. All these patterns have an impact in the context of interpersonal relationships.

Keywords: masculinities, men, social changes, gender role transition.

\footnotetext{
*Nota de las autoras:

Agradecemos a los hombres que colaboraron en este estudio por el tiempo que se tomaron para responder. El presente trabajo forma parte de un proyecto de investigación que se llevó a cabo dentro del Programa de Maestría y Doctorado en Psicología de la Universidad Nacional Autónoma de México; dicho estudio se realizó gracias a la beca otorgada a la primera autora por el Consejo Nacional de Ciencia y Tecnología (Conacyt).

La correspondencia en relación con este artículo debe dirigirse a Deysy Margarita Tovar-Hernández, Edificio C, Facultad de Psicología, unAm. Av. Universidad 3004. Col. Copilco-Universidad. C. P. 04510. Delegación Coyoacán. México D. F. Tel. 52 (55) 56222353. Dirección electrónica: psic.margaritatovar@gmail.com
} 


\section{INTRODUCCIÓN}

El modelo masculino occidental está representado predominantemente por un ideal de hombre cuyas características suelen traducirse en: joven, caucásico, fuerte desde el punto de vista físico, inteligente, potente sexualmente, exitoso en el aspecto económico, etc. A partir de este conjunto de rasgos y expectativas se gesta un modelo hegemónico de la masculinidad que se coloca en el imaginario social como un ideal al que el grupo de los hombres "deberá" adherirse (Chesebro \& Fuse, 2001; Connell, 2003; Mahalik et al., 2003), independientemente de que sea posible alcanzarlo.

En México, Díaz-Guerrero realizó estudios a lo largo de 35 años, identificando que, en el marco de las normas socioculturales que forman parte de la idiosincrasia mexicana, durante mucho tiempo prevalecieron dos premisas fundamentales: la supremacía del hombre en relación con la mujer traducida en esta visión del "padre de familia" como superior, además de una mirada sobre la "madre" como sumisa y abnegada (Díaz-Guerrero, 2003). En las premisas identificadas por el autor para conocer la psicología del mexicano, se observa que son enunciados que dan cuenta de las relaciones de género desiguales a partir de las construcciones socioculturales en torno a las diferencias biológicas.

En el caso de las normas sociales que rigen la vida de los varones, éstas versan alrededor del modelo hegemónico de la masculinidad, que en México se traduce en una forma de ser hombre que busca la subordinación de las mujeres (Díaz-Guerrero, 2003), así como la subordinación de otros hombres que no se apeguen a dicho modelo (Montesinos, 2007). Esta relación de dominación-subordinación se legitima a través de diversas instituciones, como son el Estado, la Iglesia, la escuela y la familia. Sin embargo, la masculinidad hegemónica está en un proceso de transformación ante los cambios ideológicos y estructurales que se manifiestan en los roles de género (Rocha \& Díaz-Loving, 2011), de varones y mujeres, por lo que se plantean nuevas formas de relacionarse inter e intrasexualmente (Burin, 2007; Gómez, 2007).

Las bases sobre las que se erige la masculinidad están siendo cuestionadas en la actualidad, por lo que se dice que ésta se encuentra en crisis. De acuerdo con diversos autores (Burin, 2007; Carabí, 2000; Connell,
2003; Olavarría, 2003; Seidler, 2006), dicha crisis se deriva de diferentes procesos sociales. Por una parte, dentro de los factores estructurales puede mencionarse la aplicación del modelo neoliberal en las políticas públicas, y por lo tanto, los acuerdos con los organismos internacionales, los cuales han traído como consecuencia la precarización y flexibilización laboral, así como el desempleo. Estos factores han fungido como ejes rectores de la configuración de las masculinidades hegemónicas, en tanto se mide la hombría a partir del papel de proveedor económico del varón y de su participación en el entorno laboral (Burin, 2007; Jiménez, 2007; Seidler, 2006). De esta manera, el creciente desempleo y el hecho de que las mujeres tengan ahora una mayor participación dentro del ambiente laboral, y puedan incluso fungir como proveedoras, conlleva a un cuestionamiento de este eje identitario.

Aunado a lo anterior, los movimientos sociales surgidos a partir de la segunda mitad del siglo pasado cuestionan las condiciones de desigualdad y de inequidad entre las personas por diferencias raciales, étnicas, de sexo o preferencias sexuales y demandan cambios en las asignaciones de espacios sociales (Carabí, 2000). Uno de los cuestionamientos más importantes ha sido en torno a la distribución tradicional de roles, tareas y espacios que asocian el trabajo reproductivo a las mujeres y el productivo a los hombres como una forma "natural" de relacionarse.

En la medida en la que se ha ido desmantelado esta "naturalización" de los roles de género y sus respectivas implicaciones, no sólo se ha dado lugar a un aumento en la inserción de las mujeres a los espacios públicos sino que además se puede observar el incremento de la participación en el trabajo extradoméstico remunerado durante las últimas tres décadas (Rendón, 2003) y se ha replanteado el papel que los hombres deberían jugar en el marco de dichos reacomodos.

La llamada crisis de la masculinidad también ha sido objeto de interés en los estudios de los hombres; los teóricos debaten el carácter estático y universal del concepto de la masculinidad. En Australia, Connell (2003) propuso el constructo de masculinidades para poder estudiar las transiciones de la masculinidad, ya que con esta premisa es posible reconocer su carácter dinámico y diverso, lo que ha permitido comprender que existen muchas formas de vivirse y pensarse como 
hombre, y que incluso hay diferencias hacia adentro del mismo grupo (Carabí, 2000; Collin, 2007; Jiménez, 2003). Dicho de otra forma, se deconstruye la idea de una masculinidad "inherente" al individuo.

La configuración de las masculinidades propuesta por Connell (2003) toma su referente del modelo imperante en la mayoría de los países europeos y en Estados Unidos, y se caracteriza predominantemente por la búsqueda del poder económico y político. No obstante, surgió la necesidad de reconocer que las formas en la que se viven las masculinidades pueden diversificarse en función de los entornos culturales (Hernández, 2007; Jiménez, 2003), lo que hace fundamental recurrir siempre a investigaciones de corte etnológico (Díaz-Guerrero, 2003), priorizando la idiosincrasia y subjetividades particulares de dicho contexto.

De acuerdo con esta idea de que no existe una forma única de ser hombre, algunos autores han dado cuenta de los diferentes acomodos en el proceso de configurar las masculinidades; por ejemplo, Montesinos (2007) reconoce en sus estudios nueve formas de ser hombre que transitan en un ir y venir del referente de la masculinidad hegemónica y que se configuran dentro del contexto mexicano, a partir de las transformaciones de las relaciones interpersonales, específicamente con las mujeres; por otra parte, Collin (2007) propone una matriz de rasgos prototípicos para diferenciar a las masculinidades con base en un área o aspecto de la vida de los hombres y en el cruce con la adherencia o alejamiento de los preceptos de la masculinidad tradicional.

El abordaje de los y las teóricas en la comprensión e identificación de las masculinidades reconoce la existencia de un modelo que toma como eje la masculinidad tradicional; sin embargo, permite reconocer también la diversidad existente en las formas en que los hombres viven, reconstruyen, se alejan o se adhieren a dicho modelo.

No obstante, la mayoría de las masculinidades propuestas por los investigadores anteriormente citados, suelen reducir la descripción a un solo aspecto de la vida de los hombres. Vale la pena insistir que en la noción actual de las masculinidades, las transformaciones del modelo predominante cruzan numerosas áreas y aspectos de la vida de los hombres.
La investigadora argentina Mabel Burin (2007) realizó un trabajo con parejas en el que analizó la transición que se está dando en el contexto social alrededor de los roles de género. Lo interesante de esta propuesta es que la autora reconoce el impacto que esto tiene en la construcción de las subjetividades de hombres y mujeres, así como en sus relaciones. En dicha investigación, Burin reconoce distintos espacios-momentos en las formas en que se relacionan las parejas; hay algunas que se siguen situando en los roles tradicionales de acuerdo con la división sexual del trabajo: los hombres en el espacio público y las mujeres en el doméstico. Otras, en cambio, dan cuenta del vaivén entre lo tradicional y buscan nuevas maneras de relacionarse, siendo transicionales. Burin identificó parejas que en su diario vivir intentan nuevas prácticas de convivencia más equitativas y formas innovadoras de relacionarse. Por último, hay quienes viven formas contraculturales de relacionarse y se caracterizan por una inversión en los roles tradicionales.

Este trabajo resulta de interés para la presente investigación debido a que reconoce el dinamismo que existe en la transformación de los roles de género y da cuenta de los distintos posicionamientos que se pueden tener en el proceso de vivirse como hombres y como mujeres.

La pretensión de las autoras en este artículo es comprender el posicionamiento de los hombres en las diversas maneras de vivir las masculinidades en tanto momentos críticos en constante resignificación. El propósito de este estudio fue, por lo tanto, examinar las diferentes formas de ser hombre que son reconocidas por varones de la Ciudad de México y analizar cómo dichas masculinidades pueden ser entendidas desde los espacios-momentos (tradicionales, transicionales, innovadores y contraculturales) propuestos por Burin (2007).

\section{MÉTODO}

\section{Participantes}

La selección de los participantes se llevó a cabo de forma accidental y propositiva; se contó con la colaboración de 50 hombres, con un promedio de edad de 27.8 años (rango de edad de 20 a 34 años, moda $=22$ años); todos residentes de la Ciudad de México. Para tener una visión más detallada de las características sociodemográficas de los participantes, ver la tabla 1. 
Tabla 1. Características sociodemográficas de los participantes

\begin{tabular}{|c|c|c|c|c|c|c|c|c|}
\hline \multirow{2}{*}{$\begin{array}{c}\text { Edo. } \\
\text { civil }\end{array}$} & Escolaridad & \multicolumn{7}{|c|}{ Ocupación } \\
\hline \multirow{4}{*}{ Solteros } & Empleado & Profesionista & Comerciante & Docente & Estudiante & Desempleado & Empresario \\
\cline { 2 - 10 } 42 & $\begin{array}{c}\text { Secundaria } \\
3\end{array}$ & 2 & 0 & 1 & 0 & 0 & 0 & 0 \\
\cline { 2 - 10 } & $\begin{array}{c}\text { Preparatoria } \\
22\end{array}$ & 5 & 0 & 2 & 0 & 15 & 0 & 0 \\
\cline { 2 - 10 } & $\begin{array}{c}\text { Licenciatura } \\
16\end{array}$ & 3 & 2 & 0 & 3 & 4 & 3 & 1 \\
\cline { 2 - 10 } & $\begin{array}{c}\text { Posgrado } \\
1\end{array}$ & 0 & 1 & 0 & 0 & 0 & 0 & 0 \\
\hline \multirow{2}{*}{$\begin{array}{c}\text { Total } \\
8\end{array}$} & $\begin{array}{c}\text { Secundaria } \\
1\end{array}$ & 1 & 0 & 0 & 0 & 0 & 0 & 0 \\
\cline { 2 - 10 } & $\begin{array}{c}\text { Preparatoria } \\
3\end{array}$ & 3 & 0 & 1 & 0 & 0 & 0 & 0 \\
\cline { 2 - 10 } & $\begin{array}{c}\text { Licenciatura } \\
4\end{array}$ & 1 & 1 & 0 & 0 & 1 & 0 & 0 \\
\hline
\end{tabular}

En la tabla anterior se puede observar que existe una diversidad de los participantes en los diferentes niveles de escolaridad y de ocupación, aunque cabe aclarar que un $80 \%$ reportaron ser solteros al momento de la investigación.

\section{Instrumento}

Se utilizó un cuestionario de preguntas abiertas conformado por tres apartados; en el primero se expuso el objetivo de la investigación, se garantizó la confidencialidad y el anonimato de los datos obtenidos. El segundo se conformó por dos preguntas: a) ¿Qué tipos de hombre podría identificar en México?, y b) Describa las características de los tipos de hombre que identificó. En el último se solicitaron los datos sociodemográficos como la edad, el estado civil, la escolaridad y la ocupación.

\section{Procedimiento}

Los colaboradores respondieron el cuestionario, de forma individual y a mano, en los lugares públicos donde fueron contactados. Se les solicitó su consentimiento para incorporarse al estudio. Una vez recolectados los cuestionarios se siguió el proceso de la transformación de la información en los métodos cualitativos propuestos por Castro (2002). La información obtenida se transcribió en un texto (formato RTF), para después conformar categorías que fueran representativas de la información aportada por los participantes. El análisis de contenido se realizó con el programa de análisis cualitativo Atlas ti, mediante el cual se identificaron, en los discursos, las formas de ser hombre que ellos mismos reconocen, así como las características que las conforman. Finalmente se realizó un análisis interpretativo del contenido de dichas formas de ser, y se observaron los puntos que conectaban entre sí, tomándose como ejes articuladores los diferentes espaciosmomentos de las subjetividades de género propuestos por Burin (2007).

\section{RESULTADOS}

En primer lugar, las autoras identificaron que los participantes sí reconocen diversas formas de ser hombre, ya que dieron nomenclaturas y describieron las carac- 
terísticas que las conforman. (Ver tabla 2). Los participantes nombraron masculinidades cuyas características giran en torno a los mandatos de la masculinidad hegemónica, como son: machistas, conservadores, trabajadores y padres de familia, aunque hacia adentro tienen diferencias entre aspectos positivos y negativos en términos de la deseabilidad social, contienen aspectos de roles de género masculinos altamente estereotipados.

Tabla 2. Formas de ser hombre y características que dan cuenta de las masculinidades percibidas por hombres de la Ciudad de México

\begin{tabular}{|c|c|c|}
\hline Nomenclaturas & Ejemplos de descripción & $f^{*}$ \\
\hline Machistas & Dominan a las mujeres, les gusta que se haga su voluntad cuando ellos dicen. & 15 \\
\hline Irresponsables & Machistas, borrachos y altaneros. & 4 \\
\hline Macho & Lo que dice y lo que quiere es lo que se hace y obtiene. & 2 \\
\hline Tradicionales & Machistas. & 1 \\
\hline Bárbaros & Es por esencia machista, agresivo. & 1 \\
\hline Conservador & Machista, agresivo, fuerte, violento y competitivo. & 1 \\
\hline Mujeriego & Cambia de mujer sólo por placer. & 1 \\
\hline Trabajadores & "Responsables, de buena razón”, “Buscan un asentamiento económico." & 11 \\
\hline Responsables & $\begin{array}{l}\text { "Atiende bien a su familia y provee completamente a ésta", "Se dedican a su trabajo } \\
\text { y son hombres muy cerrados." }\end{array}$ & 5 \\
\hline Padre de familia & Ejemplar, exitoso en su casa y en el trabajo. & 1 \\
\hline Contemporáneos & En transición, busca su propia manera de ser hombre. & 1 \\
\hline Posmodernos & Le dicen que sí al género pero en realidad no les importa mucho. & 1 \\
\hline Neutrales & Equilibrio entre el ideal y el mandilón. & 1 \\
\hline Abiertos & Pero tienen el control. & 1 \\
\hline Actor & $\begin{array}{l}\text { Muestra el comportamiento del hombre moderno pero su esencia sigue siendo el del } \\
\text { hombre bárbaro. }\end{array}$ & 1 \\
\hline Ideales & Fiel, simpático, responsable, buen compañero. & 2 \\
\hline Sensibles & Expresan sus emociones, son cariñosos. & 2 \\
\hline Solidarios & Género, cultura trabajada con las mujeres, con el medio ambiente. & 1 \\
\hline Maduros & Cumple con responsabilidades, le dedican tiempo a sus hijos y esposas. & 1 \\
\hline Masculinos & Seguros de sí mismos, capaces de compartir responsabilidades. & 1 \\
\hline Modernos & $\begin{array}{l}\text { Que busca el equilibrio de género y lo acepta. (Más cultura y entendimiento del } \\
\text { entorno, cooperativo socialmente). }\end{array}$ & 1 \\
\hline Mandilones & $\begin{array}{l}\text { "Sumiso", "Vive bajo las faldas de una mujer, trabaja, vive y hace todo lo posible para } \\
\text { quedar bien con su pareja." }\end{array}$ & 6 \\
\hline Humildes & Algunos abusan de ellos. & 3 \\
\hline Conformista & $\begin{array}{l}\text { Es aquel que está a gusto con lo que le da la vida, conforme le llegue, no se prepara, } \\
\text { solo espera que Dios y la vida le den para vivir. }\end{array}$ & 2 \\
\hline Mediocre & El que no está conforme pero no hace nada, el que deja que la vida pase sin más. & 2 \\
\hline
\end{tabular}

*Este dato corresponde al número de hombres que mencionaron esta forma de ser hombre. 
Sin embargo, también aluden a otras formas de vivirse como hombres, algunas de las cuales reconocen un proceso de transición en donde coexisten elementos de la "vieja guardia" y al mismo tiempo incorporan acciones como el involucramiento en la crianza de los hijos o las labores domésticas, o bien, aquellas formas que se contraponen por completo a la visión "tradicional" sobre las masculinidades. (Ver tabla 2).

De acuerdo con las frecuencias en que los participantes mencionaron las formas de ser hombre resulta cualitativamente significativo que fueron las masculinidades que comparten características tradicionales (41) las que fueron más nombradas, en comparación con el resto.

Es necesario puntualizar que los participantes describieron maneras de ser hombre atravesadas por la clase social y es importante resaltarlas debido a su significancia cualitativa (por ejemplo: burgueses, obreros, ricos y pobres). La clase social es una categoría que se entrecruza con las construcciones de género, por lo que se presentan diferentes matices que resultan de las intersecciones entre las condiciones socioeconómicas con los momentos en los que pueden situarse las masculinidades anteriormente descritas. Asimismo, los participantes reconocen masculinidades derivadas de las diferencias en las preferencias sexuales (ejemplos: gays y banda homosexual).

Otros términos fueron mencionados sin ser detallados, tales como: políticos, intelectuales, gandallas, buena onda, darketo, rokero y fiestero, aspectos que en conjunto más que características de las masculinidades pueden considerarse como ocupaciones, rasgos de personalidad o intereses musicales que no en todos los casos coinciden con el estereotipo.

Siguiendo con el análisis para tratar de identificar de qué manera estas formas de ser hombre podrían colocarse dentro de una mirada más dinámica que implique posicionarse en diferentes momentos-espacios, se pudo identificar lo siguiente:

Las formas tradicionales de ser hombre que se observan en los discursos de los varones de este estudio están representadas en mayor medida con características negativas, como ser machistas, agresivos, borrachos, dominantes con las mujeres y que les gusta que se haga su voluntad. Sin embargo, es importante puntualizar que en las respuestas los varones reconocen características positivas, como ser trabajadores, responsables, padres de familia, etc., que también se pueden situar en lo tradicional. (Ver tabla 3 ).

Tabla 3. Momentos-espacios críticos en las formas de ser hombre tomando como base la propuesta de Burin (2007)

\begin{tabular}{|l|l|c|}
\hline $\begin{array}{l}\text { Momentos- } \\
\text { espacios críticos }\end{array}$ & \multicolumn{1}{|c|}{ Elementos que permitieron identificar estas posiciones } & \multicolumn{1}{c|}{$\begin{array}{c}\text { Masculinidades } \\
\text { representativas }\end{array}$} \\
\hline Tradicionales & $\begin{array}{l}\text { Los discursos de los participantes reflejan una prevalencia de los } \\
\text { mandatos rectores de la masculinidad hegemónica (p.e. ser fuerte, } \\
\text { agresivo, etc.). }\end{array}$ & $\begin{array}{c}\text { Machistas, irresponsables, } \\
\text { tradicionales, bárbaros, } \\
\text { etc. }\end{array}$ \\
\hline Transicionales & $\begin{array}{l}\text { Se observan en las definiciones dadas por los hombres un gran número } \\
\text { de características tradicionales, a la vez que incorporan atributos de las } \\
\text { formas innovadoras y contraculturales de relacionarse. }\end{array}$ & $\begin{array}{c}\text { Contemporáneos, } \\
\text { abiertos, actor, etc. }\end{array}$ \\
\hline Innovadores & $\begin{array}{l}\text { En las respuestas dadas por los varones de este estudio se pueden } \\
\text { identificar aspectos derivados de los procesos de cambio en } \\
\text { la vida de las mujeres en específico, y los cambios globales en } \\
\text { general, generando formas más equitativas de relacionarse intra e } \\
\text { intersexualmente. }\end{array}$ & $\begin{array}{c}\text { Solidarios, maduros, } \\
\text { modernos, etc. }\end{array}$ \\
\hline Contraculturales & $\begin{array}{l}\text { Al analizar los discursos de los participantes se identificaron formas } \\
\text { de ser hombre cuyas particularidades se pueden situar como } \\
\text { las masculinidades se asocian a comportamientos de sumisión en sus } \\
\text { diferentes relaciones, primordialmente en las de pareja, estos atributos } \\
\text { denotan dificultades en la toma de decisiones de aspectos compartidos } \\
\text { en el hogar y en lo económico. }\end{array}$ & $\begin{array}{c}\text { Mandilones, humildes, } \\
\text { conformistas, etc. }\end{array}$ \\
\hline
\end{tabular}


En el análisis de la información, las autoras identificaron formas transicionales que aluden precisamente a la coexistencia de aspectos tradicionales, innovadores y contraculturales. En las descripciones de las masculinidades, nombradas como: contemporáneos, neutrales, abiertos, etc., dadas por los participantes de este estudio, se observa el proceso de transición, es decir el ir y venir entre la incorporación de aspectos tradicionales ligados con la "hombría" (fuerza, dominio, caballerosidad y rol proveedor) y de características expresivas, que en algunos casos se identifican con roles asociados con el estereotipo de lo "femenino". (Ver tabla 3).

En la tabla 3 se puede observar el acomodo de las formas de ser hombre identificadas por los colaboradores, como ideales, sensibles, modernos, entre otros, en cuyas definiciones se observan maneras innovadoras y más equitativas de los varones para relacionarse, como son compartir responsabilidades, expresión emocional, seguridad en sí mismos, ser cooperativos socialmente y "buscar el equilibrio de género".

Por otra parte, al analizar los discursos de los participantes se identificaron formas de ser hombre cuyas particularidades se pueden situar como momentos contraculturales que coexisten con las otras masculinidades; las denominaciones dadas por los participantes fueron: sumisos, mediocres, mandilones y conformistas. Las características enlistadas se sitúan en contracorriente con lo que se espera socialmente del "deber ser" de los hombres, como por ejemplo, se reconocen comportamientos como el dejarse mangonear por sus parejas y permitir que los demás abusen de ellos. (Ver tabla 3 ).

\section{DISCUSIÓN}

Los participantes de este estudio reconocieron diferentes formas de ser hombre, con lo que se puede inferir que existe un proceso de deconstrucción de la masculinidad "hegemónica", lo que cuestiona precisamente el carácter estático y universal de sus supuestos y hace evidente que las masculinidades forman parte de procesos inacabados y dinámicos (Connell, 2003).

Los hombres que colaboraron en esta investigación describen características de las masculinidades que se pueden situar en diferentes estadios del proceso de transición del ideal masculino (Collin, 2007; Jiménez, 2003), acercándose o alejándose de éste y reconocen aspectos que pueden situarse en momentos más tradicionales, transicionales, innovadores y/o contraculturales, tomando como base el análisis realizado por Burin (2007).

Es notable que en las descripciones de los participantes predominan numéricamente formas de ser hombre cuyas características se pueden ubicar en momentos tradicionales de relacionarse; es decir, en la ideología masculina sigue imperando el modelo hegemónico de que los hombres en México se caracterizan por ser machistas, agresivos, mujeriegos, borrachos y dominantes, lo que coincide con la literatura (DíazGuerrero, 2003; Montesinos, 2007).

En los discursos de los participantes se pueden observar aspectos positivos, en términos de deseabilidad social, como ser responsables, trabajadores, competitivos y proveer económicamente. Esto es fundamental, ya que devela que en el proceso de las construcciones de la masculinidad pueden coexistir formas tradicionales de dominación, con dos ejes que van y vienen de lo deseable y aceptado socialmente, hasta características poco deseables; sin embargo, en ambos casos se tratan de características muy estereotipadas.

Siguiendo con la discusión, el predominio de características apegadas al modelo tradicional remarca la dificultad para generar esquemas cognoscitivos mucho más flexibles (Carabí, 2000), entre otras cosas porque el contenido de estos se coloca como un eje fundamental en el ejercicio de conformar identidades sociales y colectivas. Sin embargo, es necesario reconocer que en el imaginario colectivo parecen convivir diferentes formas de vivirse como hombres, de ahí que surjan otros acomodos o configuraciones en momentos críticos de cambio social.

Por ejemplo, en el reconocimiento que los hombres de este estudio dan a ciertas características de las masculinidades que se pueden situar como formas transicionales, se agruparon nomenclaturas que en apariencia buscan relaciones equitativas, pero que continúan realizando conductas de dominación, principalmente hacia las mujeres (Montesinos, 2007). En particular llama la atención aquí el hecho de que las transformaciones de las masculinidades no se dan en un solo sentido, hacia la equidad, sino que pueden adoptar modos sutiles de dominación. Esto podría analizarse desde los mecanismos y estrategias de resistencia y oposición que se pue- 
den generar hacia una transición, la que en el caso de los varones implica precisamente la pérdida de ciertos privilegios según el modelo patriarcal (Cobo, 2011).

Los aspectos que se pueden situar como maneras innovadoras reconocidas por los hombres de este estudio, parecen aludir a una postura flexible y abierta hacia la equidad; se muestran atributos como compartir responsabilidadeseconómicas, el cuidado deloshijos, tener una mayor expresión emocional, etc., estas son masculinidades emergentes que surgen dentro de un contexto de transición social (Rocha \& Díaz-Loving, 2011).

Finalmente, en el discurso de los hombres se observan aquellos elementos de las masculinidades que se pueden acomodar como momentos contraculturales, como ser sumisos y realizar conductas asociadas a la feminidad. Se observan características referentes al conformismo y a la mediocridad, con lo cual transgreden uno de los mandatos principales del modelo occidental de la masculinidad, que es la búsqueda incesante del éxito (Chesebro \& Fuse, 2001). Estas masculinidades contraculturales parecen ser formas invertidas de dominación (Burin, 2007), donde son ellos quienes se encuentran en una postura de sumisión y vulnerabilidad en los diferentes contextos, gestándose una inversión de los roles, pero no existe un cuestionamiento sobre la disparidad y la asimetría existente entre los sexos. Sin embargo, es importante señalar que aludir a estas manifestaciones como una inversión de la situación de dominación, y en muchos casos como una descalificación hacia los varones, refleja la importancia que tiene contextualizar estos hallazgos en medio de las relaciones de poder y el androcentrismo que coacciona no sólo la configuración, sino las relaciones entre los géneros, de tal suerte que cuando los varones "abandonan" -por decirlo de alguna manera- los roles "tradicionales" e incursionan en otros no tradicionales -equívocamentepensados como femeninos, se cuestiona su hombría y se devalúan sus acciones, por lo que habría que analizar con mayor profundidad estos hallazgos.

Una conclusión importante del presente trabajo es precisamente el reconocimiento de que bajo estas formas de masculinidad se pueden dar diferentes matices, es decir los momentos por los que transitan las masculinidades no son excluyentes entre sí, de tal cuenta que pueden coexistir ciertos aspectos tradicionales a la vez que pueden situarse como contraculturales, dependiendo de los espacios de interacción social, como son la familia, trabajo, relaciones de pareja, entre otros.

Es necesario reconocer que los momentos críticos en los que se pueden situar las masculinidades son consecuencia de un proceso dinámico, resultante de la interacción de los sucesos globales y locales, por lo que no podemos dejar de lado que existen modelos occidentales que ejercen influencia en las formas en que se construyen las subjetividades, pero es importante analizar las peculiaridades regionales en las que se erigen (Hernández, 2007), por lo que es recomendable que se sigan realizando investigaciones e intervenciones desde las diferentes ciencias sociales de corte etnológico (Díaz-Guerrero, 2003).

Los hallazgos aquí presentados se derivan del análisis de una parte del proceso de reconfiguración de las masculinidades dentro de un escenario social cambiante en el contexto de la Ciudad de México. 


\section{REFERENCIAS}

Burin, M. (2007). Trabajo y parejas: impacto del desempleo y de la globalización en las relaciones entre los géneros. En M. Jiménez \& O. Tena (Coords.), Reflexiones sobre masculinidades y empleo (pp. 5980). México: Centro Regional de Investigaciones Multidisciplinarias, Universidad Nacional Autónoma de México.

Carabí, A. (2000). Construyendo nuevas masculinidades: una introducción. En M. Segarra \& A. Carabí (Eds.), Nuevas masculinidades (pp. 15-27). Barcelona: Icaria.

Castro, R. (2002). En busca del significado: supuestos, alcances y limitaciones del análisis cualitativo. En I. Szasz \& S. Lerner (Comps.), Para comprender la subjetividad (pp. 57-85). México: Centro de Estudios Demográficos y de Desarrollo Urbano, El Colegio de México.

Chesebro, J. \& Fuse, K. (2001). The Development of a Perceived Masculinity Scale. Communication Quarterly, 3(49), 203-278.

Cobo, R. (2011). Hacia una nueva política sexual. Las mujeres ante la reacción patriarcal. Madrid: Catarata.

Collin, L. (2007). Masculinidades: aportes para su clasificación. En M. Jiménez \& O. Tena (Coords.), Reflexiones sobre masculinidades y empleo (pp. 205233). México: Centro Regional de Investigaciones Multidisciplinarias, Universidad Nacional Autónoma de México.

Connell, R. W. (2003). Masculinidades. México: Programa Universitario de Estudios de Género.

Díaz-Guerrero, R. (2003). Bajo las garras de la cultura. Psicología del mexicano 2. México: Trillas.

Gómez, M. (2007). Masculinidad en la "Sociedad de riesgo". En M. Jiménez \& O. Tena (Coords.), Reflexiones sobre masculinidades y empleo (pp. 33-57). México: Centro Regional de Investigaciones Multidisciplinarias, Universidad Nacional Autónoma de México.

Hernández, O. (2007). Estudios sobre masculinidades. Aportes desde América Latina. Revista de Antropología Experimental, 7, 153-160.
Jiménez, M. (2003). Dando voz a los varones. Sexualidad, reproducción y paternidad de algunos mexicanos. México: Centro Regional de Investigaciones Multidiciplinarias, Universidad Nacional Autónoma de México.

Jiménez, M. (2007). Algunas ideas acerca de la construcción social de las masculinidades y las feminidades, el mundo público y el mundo privado. En M. Jiménez \& O. Tena (Coord.), Reflexiones sobre masculinidades y empleo (pp. 99-118). México: Centro Regional de Investigaciones Multidisciplinarias, Universidad Nacional Autónoma de México.

Mahalik, J., Ludlow, L., Diemer, M., Locke, B., Scott, R. J., Gottfried, M. \& Freitas, G. (2003). Development of the Conformity to Masculine Norms Inventory. Psychology of Men \& Masculinity, 4(1), 3-25.

Montesinos, R. (2007). Ensayando sobre nuevas tipologías de la masculinidad. En M. Jiménez \& O. Tena (Coords.), Reflexiones sobre masculinidades y empleo (pp. 181-204). México: Centro Regional de Investigaciones Multidisciplinarias, Universidad Nacional Autónoma de México.

Olavarría, J. (2003). Los estudios sobre masculinidades en América Latina. Anuario Social y Político de América Latina y el Caribe, 6, 91-98.

Rendón, T. (2003). Trabajo de hombres y trabajo de mujeres en el México del siglo XX. México: Programa Universitario de Estudios de Género, Centro Regional de Investigaciones Multidisciplinarias, Universidad Nacional Autónoma de México.

Rocha, T. \& Díaz-Loving, R. (2011). Identidades de género. Más allá de cuerpos y mitos, México: Editorial Trillas.

Seidler, V. (2006). Masculinidades, hegemonía y vida emocional. En G. Careaga \& S. Cruz (Coords.), Debates sobre Masculinidades (pp. 147-157). México: Programa Universitario de Estudios de Género. 